\title{
Role of Insulin Sensitizers in Raised Alanine Aminotransferase in Non-alcoholic Fatty Liver Disease in Glucose Intolerance Patients: A Short-Term Experience with Metformin Plus Pioglitazone Versus Metformin Alone
}

\author{
SR SULTANA ${ }^{\mathrm{a}}$, MF AMIN ${ }^{\mathrm{b}}$, MA RAHIM ${ }^{\mathrm{c}}$, MM RAHMAN ${ }^{\mathrm{d}}$, MN HOQUE ${ }^{\mathrm{e}}$, TM BHUIAN ${ }^{\mathrm{f}}$, \\ IK DATTA'g, MA RAHMAN ${ }^{\text {h }}$
}

Summary:

Objectives:To evaluate and compare the effectiveness of metformin plus pioglitazone versus metformin alone in treatment of raised alanine aminotransferase (ALT) in nonalcoholic fatty liver disease (NAFLD) in patients with newly detected diabetes mellitus (DM) and impaired glucose tolerance (IGT).

Materials and methods: In this open label clinical trial, newly detected DM and IGT patients with raised ALT and ultrasound proven NAFLD were treated with either

a. Dr. Syeda Rezina Sultana, Senior Medical Officer, OPD, BIRDEM, Shahbag, Dhaka.

b. Dr. Mohammod Feroz Amin, Assistant Professor, Department of Endocrinology, Ibrahim Medical College and BIRDEM General Hospital, Shahbag, Dhaka.

c. Dr. Muhammad Abdur Rahim, Registrar, Department of Internal Medicine, Ibrahim Medical College and BIRDEM General Hospital, Shahbag, Dhaka.

d. Dr. Md. Mahbubur Rahman, Senior Medical Officer, Department of GastroIntestinal, Hepatobiliary and Pancreatic Diseases, BIRDEM General Hospital, Shahbag, Dhaka.

e. Dr. Md. Nazmul Hoque, Junior Consultant, Department of GastroIntestinal, Hepatobiliary and Pancreatic Diseases, Ibrahim Medical College and BIRDEM General Hospital, Shahbag, Dhaka.

f. Dr. Tareq Mahmud Bhuian, Associate Professor, Department of GastroIntestinal, Hepatobiliary and Pancreatic Diseases, Ibrahim Medical College and BIRDEM General Hospital, Shahbag, Dhaka.

g. Dr. Indrajit Kumar Datta, Registrar, Department of GastroIntestinal, Hepatobiliary and Pancreatic Diseases, Ibrahim Medical College and BIRDEM General Hospital, Shahbag, Dhaka.

h. Prof. Md. Anisur Rahman, Professor, Department of GastroIntestinal, Hepatobiliary and Pancreatic Diseases, Ibrahim Medical College and BIRDEM General Hospital, Shahbag, Dhaka.

Address of Correspondance: Dr. Mohammod Feroz Amin, Assistant Professor, Department of Endocrinology, Ibrahim Medical College and BIRDEM General Hospital, Room NO 1537, 122, KaziNazrul Islam Avenue, Shahbag, Dhaka-1000, Bangladesh, Phone: 01715010102, 01741009000, E-mail: feroz_amin@yahoo.com, muradrahim23@yahoo.com

Received: 21 September, 2013

Accepted: 20 April, 2014 metformin and pioglitazone combination (group 1) or metformin alone (group 2). They were followed up upto 6 months.

Results: Total number of patients was 49 (27 in group 1 and 22 in group 2) and there was male predominance in either group. Age was almost identical between two groups (46 \pm 9.3 and 45.4 \pm 5.7 years in group 1 and group 2 respectively). Significant reduction in values of fasting blood glucose (FBG), 2 hours post breakfast values (ABF), HbA1c, cholesterol (CHOL), triglycerides (TG) and ALT of the study subjects were achieved in either group after six months (Group 1: FBG 8.89 \pm 1.4 vs 6.37 $\pm 0.5 \mathrm{mmol} / \mathrm{l}, \mathrm{ABF} 13.2 \pm 2.07$ vs $8.34 \pm 0.84 \mathrm{mmol} / \mathrm{l}, \mathrm{HbA1c} 8.15 \pm 0.87 \%$ vs6.7 $\pm 0.40 \%$, CHOL 205.26 \pm 30.74 vs $178.89 \pm 18.59 \mathrm{mg} / \mathrm{dl}, \mathrm{TG}$ $226.15 \pm 50.06$ vs $155.85 \pm 20.99 \mathrm{mg} / \mathrm{dl}$, ALT $91.52 \pm 23.14$ vs $45.74 \pm 12.63 \mathrm{mg} / \mathrm{dl}$ and in Group 2 : FBG 9.39 \pm 2.26 vs $6.98 \pm 1.20 \mathrm{mmol} / \mathrm{l}, \mathrm{ABF} 13.38 \pm 2.93$ vs $9.13 \pm 1.46 \mathrm{mmol} /$, HbA1c 8.10 $\pm 0.92 \%$ vs7.03 $\pm 0.71 \%$, CHOL 206.55 \pm 29.9 vs $195 \pm 23.55 \mathrm{mg} / \mathrm{dl}$, TG $235.59 \pm 46.22 \mathrm{vs} 178.91 \pm 38.24 \mathrm{mg} /$ dl, ALT 105.59 \pm 18.63 vs $66.36 \pm 16.02 \mathrm{mg} / \mathrm{dl}$ ). In comparison between two groups, Group 1 had better metabolic control compared to their counterpart of Group 2 at the end of 6 months [Group 1 vs Group 2: FBG $(p=0.024), A B F$ $(p=0.022), C H O L(p=0.010), T G(p=0.010)]$. There was significant reduction in ALT as well $(p=0.000)$.

Conclusion:Combination of metformin and pioglitazone is more effective than metformin alone in reducing ALT in NAFLD in newly detected DM and IGT patients.

Key words: Alanine aminotransferase, diabetes mellitus, impaired glucose tolerance, non-alcoholic fatty liver disease.

(J Banagladesh Coll Phys Surg 2014; 32: 194-199)

Introduction:

The epidemics of obesity, metabolic syndrome, type 2 diabetes mellitus (DM) and atherosclerosis are increasing worldwide. ${ }^{1}$ Non-alcoholic fatty liver disease (NAFLD), long time unnoted entity, is becoming recognized as a condition possibly involved in the 
pathogenesis of these diseases. Support for this hypothesis emerges from studies revealing that NAFLD precedes the manifestations of the metabolic derangements. ${ }^{2,3}$ The spectrum of NAFLD may be from simple hepatic steatosis to non-alcoholic steatohepatitis (NASH) which may be complicated to cirrhosis and even hepatocellular carcinoma. ${ }^{4,5}$

The term NAFLD is used to describe a condition of fat accumulation in liver in absence of excessive alcohol consumption ( $<20 \mathrm{~g} /$ day) and other specific causes of hepatic steatosis. NAFLD affects some $10-24 \%$ of general population and the prevalence increases upto 6 foldin obese persons. ${ }^{6}$ NAFLD is the cause of asymptomatic elevation of ALT in up to $90 \%$ of cases. $^{7}$

Regardless of body mass index (BMI), the presence of type 2 DM significantly increases the risk and severity of NAFLD. ${ }^{4,5}$ Trunkal obesity is an important risk factor even in a patients with a normal BMI. ${ }^{8}$ About half the patients with hyperlipidemia are found to have NAFLD on ultrasonography (USG). ${ }^{9}$

Most patients with NAFLD are asymptomatic at diagnosis. Common symptoms are fatigue, malaise, right upper quadrant pain or discomfort and sensation of fullness. Hepatomegaly is common. ${ }^{10,11}$ Other findings relate to obesity and other features of metabolic syndrome. Mild to moderate increased serum levels of aspartate aminotransferese (AST), ALT or both are the most common and often the only laboratory abnormality. The ratio of AST: ALT is usually $<1$ and the ratio increases as fibrosis advances. ${ }^{10}$ Hypertriglyceridemia increases the risk of NAFLD. ${ }^{9}$

Good metabolic control in DM or dyslipidaemia is not always effective in reversing NAFLD. Weight loss shows improvement on liver test results. ${ }^{12-15}$ No medications have been proved to directly reduce or reverse liver damage independent of weight loss. Many drugs have been used with variable degree of improvements in liver function tests and hepatic histology. ${ }^{16-21}$

Emerging evidence confirms that NAFLD can be progressive and associated with significant morbidity and mortality. Despite efforts aimed at improving early detection and prevention, many patients are still seen at the advanced stages. ALT is the best and most reliable non-invasive method for screening NAFLD.
Over the last few years, clinical trials evaluated the use insulin sensitizers in treatment of NAFLD. Mixed results, heterogeneous therapeutic approaches and the small numbers of subjects have limited their applications as clinical guideline. In the current study, we have tried to evaluate the effectiveness of the combined insulin sensitizers which would act on different receptors at the tissue level.

\section{Materials and Methods:}

This open level clinical trial was carried out in the outpatient (OPD) wing of Department of Gastrointestinal, Hepatobiliary and Pancreatic Diseases (GHPD) at Bangladesh Institute of Research and Rehabilitation in Diabetes, Endocrine and Metabolic Disorders (BIRDEM), Dhaka, Bangladesh in the year 2010. Sixty newly detected DM and IGT patients, referred at GHPD-OPD for raised ALT and ultrasound proven NAFLD were enrolled in this study. Patients with chronic liver disease (CLD), positive HBsAg and antiHCV and history of alcoholism were excluded from the study. All patients were on hypocaloric diet. Every alternate patients were prescribed either metformin 850 $\mathrm{mg}$ twice daily and pioglitazone $30 \mathrm{mg}$ once daily (Group 1) or metformin 850 mg twice daily (Group 2) for six months. Total 49 patients (27 patients in group 1 and 22 in group 2) completed the trial. Patients were assessed clinically and by laboratory investigations at enrolment (visit 1), at $3^{\text {rd }}$ (visit 2) and at $6^{\text {th }}$ months (visit 3). Satistical analysis was done by using frequencies and percentages and by the applying paired sample test within the group and unpaired t test and chi-square test between groups and $p$ value of $<0.05$ was considered significant. Ethical clearance was obtained from ethical review committee of BIRDEM before starting the trial and informed written consent was taken from all patients before enrolment.

\section{Results:}

Total number of patients was 49 . Mean age was $45.80 \pm 8.54$ years. Male were $65.3 \%$ and female were $34.7 \%$. Baseline and anthropometric measurements of study population were shown in Table I. Thirteen (26.5\%) patients were asymptomatic, 14 (28.6\%) patients had a feeling of abdominal fullness, 10 (20.4\%) patients complained about upper abdominal pain and 12 (24.5\%) complained about both upper abdominal pain and fullness. Twenty seven (55.1\%) patients were treated by metformin $850 \mathrm{mg}$ twice and pioglitazone 
$30 \mathrm{mg}$ once daily (Group 1) and 22 (44.9\%) patients were treated by metformin $850 \mathrm{mg}$ twice daily (Group 2).

Significant improvements were noted with treatment in metabolic control like fasting and post prandial blood glucose, HbA1c and lipid profile in both groups in $2^{\text {nd }}$ and $3^{\text {rd }}$ visits (Table II and III).
Values of ALT showed significant improvements in $2^{\text {nd }}$ and $3^{\text {rd }}$ visits (Table IV). The inter-group difference regarding ALT, FBG and TG were significant in every visit (Table V). Though the inter-group difference regarding $\mathrm{ABF}$, total cholesterol and HbA1c was not significant at $1^{\text {st }}$ visit, it became significant in subsequent visits (Table 5).

Table I

\section{Baseline characteristics and anthropometric measurements of study population}

\begin{tabular}{lcc} 
Variables & Group 1 $(\mathrm{n}=27)$ & Group 2 $(\mathrm{n}=22)$ \\
\hline Age (years) & $46.0 \pm 9.3$ & $45.4 \pm 5.7$ \\
Sex (male, female) (\%) & $74.1,25.9$ & $54.5,45.5$ \\
DM, IGT (\%) & $92.6,7.4$ & $81.8,18.2$ \\
Height (cm) & $159.9 \pm 6.1$ & $157.6 \pm 7.9$ \\
Weight (kg) & $69.4 \pm 8.5$ & $68.5 \pm 11.0$ \\
BMI (kg/m2) & $27.1 \pm 2.2$ & $27.4 \pm 3.2$ \\
Waist circumference (cm) & $90.6 \pm 4.2$ & $91.3 \pm 4.7$ \\
Hip (cm) & $93.5 \pm 3.5$ & $94.1 \pm 4.1$ \\
\hline
\end{tabular}

Table-II

\begin{tabular}{|c|c|c|c|c|}
\hline \multicolumn{5}{|c|}{ Biochemical parameters of group 1 in three visits } \\
\hline Variables & Visit & mean \pm SD & Comparison between visits & P value \\
\hline \multirow{3}{*}{$\mathrm{FBG}(\mathrm{mmol} / \mathrm{L})$} & 1 & $8.89 \pm 1.44$ & $\mathrm{~V} 1-\mathrm{V} 2$ & $<0.001$ \\
\hline & 2 & $7.04 \pm 0.79$ & V2-V3 & $<0.001$ \\
\hline & 3 & $6.37 \pm 0.50$ & V1-V3 & $<0.001$ \\
\hline \multirow{3}{*}{$\mathrm{ABF}(\mathrm{mmol} / \mathrm{L})$} & 1 & $13.20 \pm 2.07$ & $\mathrm{~V} 1-\mathrm{V} 2$ & $<0.001$ \\
\hline & 2 & $9.48 \pm 0.99$ & V2-V3 & $<0.001$ \\
\hline & 3 & $8.38 \pm 0.84$ & V1-V3 & $<0.001$ \\
\hline \multirow{3}{*}{ HbA1c (\%) } & 1 & $8.15 \pm 0.87$ & $\mathrm{~V} 1-\mathrm{V} 2$ & $<0.001$ \\
\hline & 2 & $7.20 \pm 0.47$ & V2-V3 & $<0.001$ \\
\hline & 3 & $6.70 \pm 0.40$ & V1-V3 & $<0.001$ \\
\hline \multirow{3}{*}{ CHOL (mg/dl) } & 1 & $205.26 \pm 30.74$ & V1-V2 & $<0.001$ \\
\hline & 2 & $187.44 \pm 21.58$ & V2-V3 & $<0.001$ \\
\hline & 3 & $178.89 \pm 18.59$ & V1-V3 & $<0.001$ \\
\hline \multirow{3}{*}{ TG (mg/dl) } & 1 & $226.15 \pm 50.06$ & V1-V2 & $<0.001$ \\
\hline & 2 & $177.26 \pm 26.62$ & V2-V3 & 0.004 \\
\hline & 3 & $155.85 \pm 20.99$ & V1-V3 & $<0.001$ \\
\hline
\end{tabular}

Data were expressed as maen $\pm \mathrm{SD}$. Mean values between the visits were compared by paired t-test. 


\section{Table-III}

\section{Biochemical parameters of group 2 in three visits}

\begin{tabular}{lcccc} 
Variables & Visit & mean \pm SD & Comparison between visits & P value \\
\hline \multirow{2}{*}{ FBG (mmol/L) } & 1 & $9.39 \pm 2.26$ & V1-V2 & $<0.001$ \\
& 2 & $7.88 \pm 1.53$ & V2-V3 & 0.003 \\
& 3 & $6.98 \pm 1.20$ & V1-V3 & $<0.001$ \\
ABF (mmol/L) & 1 & $13.39 \pm 2.93$ & V1-V2 & $<0.001$ \\
& 2 & $9.90 \pm 1.76$ & V2-V3 & $<0.001$ \\
HbA1c (\%) & 3 & $9.13 \pm 1.46$ & V1-V3 & $<0.001$ \\
& 1 & $8.10 \pm 0.92$ & V1-V2 & $<0.001$ \\
& 2 & $7.34 \pm 0.76$ & V2-V3 & 0.003 \\
CHOL (mg/dl) & 3 & $7.03 \pm 0.71$ & V1-V3 & $<0.001$ \\
& 1 & $206.55 \pm 29.90$ & V1-V2 & $<0.001$ \\
& 2 & $191.14 \pm 26.12$ & V2-V3 & 0.291 \\
& 3 & $195.00 \pm 23.55$ & V1-V3 & 0.034 \\
TG (mg/dl) & 1 & $235.59 \pm 46.22$ & V1-V2 & $<0.001$ \\
& 2 & $199.82 \pm 40.73$ & V2-V3 & 0.004 \\
& 3 & $178.91 \pm 38.24$ & V1-V3 & $<0.001$ \\
\hline
\end{tabular}

Data were expressed as maen \pm SD. Mean values between the visits were compared by paired t-test.

\section{Table-IV}

\section{Serum ALT levels in three visits in two groups}

\begin{tabular}{ccccc} 
Group & Visit & ALT $(\mathrm{U} / \mathrm{L})$ & Comparison between visits & P value \\
\hline \multirow{2}{*}{1} & $91.52 \pm 23.14$ & V1-V2 & $<0.001$ \\
& 2 & $62.78 \pm 14.79$ & V2-V3 & $<0.001$ \\
& 3 & $45.74 \pm 12.63$ & V1-V3 & $<0.001$ \\
& 1 & $105.59 \pm 18.63$ & V1-V2 & $<0.001$ \\
& 2 & $81.77 \pm 16.29$ & V2-V3 & $<0.001$ \\
& 3 & $66.36 \pm 16.02$ & V1-V3 & $<0.001$ \\
\hline
\end{tabular}

Data were expressed as mean \pm SD. Mean values between visits were compared by paired t-test. 
Table-V

\begin{tabular}{lcccc}
\multicolumn{5}{c}{ Significance of different biochemical parameters between two groups } \\
Variables & Visit & Group & Mean \pm SD & P value \\
\hline \multirow{2}{*}{ FBG (mmol/L) } & 1 & 12 & $8.87 \pm 1.449 .39 \pm 2.26$ & 0.338 \\
& 2 & 12 & $7.04 \pm 0.797 .87 \pm 1.52$ & 0.018 \\
& 3 & 12 & $6.37 \pm 0.556 .98 \pm 1.20$ & 0.024 \\
ABF (mmol/L) & 1 & 12 & $13.20 \pm 2.0713 .38 \pm 2.93$ & 0.805 \\
& 2 & 12 & $9.48 \pm 0.999 .90 \pm 1.75$ & 0.299 \\
HbA1c (\%) & 3 & 12 & $8.34 \pm 0.849 .13 \pm 1.46$ & 0.022 \\
& 1 & 12 & $8.15 \pm 0.848 .11 \pm 0.92$ & 0.844 \\
CHOL (mg/dl) & 2 & 12 & $7.21 \pm 0.477 .34 \pm 0.76$ & 0.459 \\
& 3 & 12 & $6.74 \pm 0.407 .03 \pm 0.71$ & 0.086 \\
& 1 & 12 & $205.26 \pm 30.74206 .55 \pm 29.30$ & 0.883 \\
TG (mg/dl) & 2 & 12 & $187.44 \pm 21.58191 .14 \pm 26.12$ & 0.590 \\
& 3 & 12 & $178.89 \pm 18.59195 .00 \pm 23.55$ & 0.010 \\
& 1 & 12 & $226.15 \pm 50.06235 .59 \pm 46.22$ & 0.500 \\
ALT (U/L) & 2 & 12 & $177.26 \pm 26.62199 .82 \pm 40.73$ & 0.024 \\
& 3 & 12 & $155.85 \pm 20.99178 .91 \pm 38.24$ & 0.010 \\
& 1 & 12 & $91.52 \pm 23.14105 .59 \pm 18.63$ & 0.026 \\
\hline
\end{tabular}

Data were expressed as mean $\pm \mathrm{SD}$. Maen values of two groups were compared by unpaired t-test.

\section{Discussion:}

Treatment strategies for NAFLD are improving the insulin resistance by weight loss, exercise and pharmacotherapy with insulin sensitizers. In our study, we have tried to evaluate and compare the efficacy of metformin and pioglitazone combination versus metformin alone for the reduction of ALT in NAFLD.

Mean age of the study population was over 45 years. In a study $65.4 \%$ patients were in 40 - 59 years age group. ${ }^{3}$ As previously noted, NAFLD is the hepatic manifestation of metabolic syndrome. Risk factors associated with NAFLD include central obesity, type2 DM, insulin resistance and dyslipidemia. ${ }^{4,23}$ The values of mean BMI, waist circumference, and TG in our patients clearly indicate that insulin resistance was risk factor for developing NAFLD in our population as well. In a population based study,it was found that waist circumference was recognized as a simple parameter for estimation of liver fat accumulation. It was found that presence of NAFLD correlates significantly with BMI and waist hip ratio which support our findings. ${ }^{6}$

Over the period of 3 and 6 months follow up, both the treatment groups showed improvements in HbA1c, more in combination group which confirmed the well established issues of glucose lowering effect of metformin and pioglitazone and more powerful blood glucose lowering effect of combination of both the drugs as stated in American Diabetic Association (ADA) guideline 2012 and guideline of American College of Clinical Endocrinology (AACE) 2012.

A reduction in ALT is an acceptable end point for a proof of concept of exploratory trial in NAFLD. In our trial, serum ALT showed significant improvement at $3^{\text {rd }}$ and $6^{\text {th }}$ months in both groups. These finding confirmed the beneficial effect of insulin sensitizers in NAFLD as seen in other studies. ${ }^{18-22}$ These studies evaluated either metformin or thiazolidinediones versus diet and lifestyle measures and no study 
evaluated combination of insulin sensitizers. Our findings are in favor of combination of drugs.

Metformin is less expensive than most other treatment modalities of NAFLD. Most studies with prolonged use of metformin have shown no or little side effects and thus can be used safely for long period of time. For pioglitazone current recommendations are that the patients should be clinically monitored and it should not be used in those with advanced congestive cardiac failure (NYHA class III and IV).

Our study had some limitations. Limited numbers of patients were evaluated for a short time. Liver biopsy was not done and patients were not followed up with fibroscan or USG.

Conclusion:

In conclusion, it can be said that both metformin and combination of metformin and pioglitazone are effective in reducing ALT in NAFLD and combination is better. Not only that, the combination group had better results in improving lipid profile and glycaemic status. However, larger, multicenter studies can be done for better and more reliable results.

\section{References:}

1. Norbert S, Konstantinos K, Hans H. Causes of Metabolic Consequences of Fatty liver. Endocrine review 2010; 29 (7):939-60.

2. Shibata M, KiharaY, Taguchi M, Tashiro M, Otsuki M. Nonalcoholic Fatty liver disease is a risk factor for type 2 diabetes in middle aged Japanese men. Diabetes Care 2007; 30:294044.

3. Targher G, Bertolini L, Poli F, et al. Prevelance of non-alcoholic fatty liver disease and risk of future cardiovascular events among type 2 diabetic patients. Diabetes Care 2007;54:354146.

4. Silverman JF, O’Berin KF, Long S, et al. Liver pathology in morbidly obese patients with and without diabetes. Am J gastroenterol 1990;85:1349-55.

5. Wanless IR, Lentz JS. Fatty liver hepatitis (steatohepatitis) and obesity: an autopsy study with analysis of risk factors. Hepatology 1990;12:1106-10.

6. Nomura H, Kashiwagi S,Hayashi J, et al. Prevalence of fatty liver in a general population of Okinawa, Japan. Jpn J Med 1998;27:142-49.

7. Daniel S, Ben Menachem T, Vasudevan G, Blumenkchl M. Prospective evaluation of unexplained chronic liver transaminase abnormalities in asymptomatic and symptomatic patients. Am j Gastroenterol 1999;94:3010-14.

8. Ruderman N, Chisholm D, Pi-Sunyer X, Schncider S. The metablically obese, normal weight individual revisited. Diabetes 1998;47:699-713.

9. Assy N, Kaita K, Mymin D, Levy C, Rosser B, Minuk G. Fatty infiltration of liver in hyperlipidemic patients. Dig Dis Sci 2000;45:1929-34.

10. Angulo P, Lindor KD. Non-alcoholic fatty liver disease. J GastroenterolHepatol 2002;17:186-90.

11. Wieckowsaka A, McCullough AJ, Feldstien AE. Noninvasive diagnosis and monitoring of non-alcoholic steatohepatitis: present and future. Hepatology 2007;46:582-89.

12. LuyckxFh, Deasive C, Thirty A, et al. Liver abnormalities in severely obese subjects: effect of drastic weight loss in gastroplasty. Int J Obsess RelatMetab Discord 1998; 22: 222-26.

13. Anderson T, Gluud C, Franzmann MB, Cristoffersen P. Hepatic effects of dietary weight loss in morbidly obese subjects. J Hepatol 1991;12:224-29.

14. Palmr M, Schaffner F. Effect of weight reduction on hepatic abnormalities in overweight patients. Gastroenterology 1990;99:1408-13.

15. Uneo T, Sugawara H, Sujaku K, et al. Therapeutic effects of restricted diet and exercise in obese patient with fatty liver. J Hepatol 1997; 27:103-107.

16. Basanough M, Acbay O, SOnsuz A. A controlled trial of gemfibrozil in the treatment of patients with non-alcoholic steatohepaitis. J Hepatol 1999;31:384-87.

17. Lavine JE. Vitamin E treatment of non-alcoholic steatohepaitis in children: A pilot study. J Pediatr 2000;136:734-38.

18. Marchesini G, Brizi M, Bianchi G, et al. Metformin in nonalcoholic steatohepatitis. Lancet 2001;358:893-98.

19. Laurin J, LindonKD,Crippin JS, et al. Urodeoxecholic acid or clofibrate in the treatment of non-alcoholic induced steatohepatitis: a pilot study. Hepatology 1996;23:1464-67.

20. Hasegawa T, Yoneda M, Nakamura K, Makino T, Terano A. Plasma transforming growth factor â 1 level and efficacy of á-tocopherol in patients with non-alcoholic steaohepatitis. AlmentPharmacolTher 2001;15:1667-72.

21. Caldwell SH, Hespenhiede EE, Redick JA, Jezzoni JC, Battle $\mathrm{EH}$, Sheppard BL. A pilot study of thiazolidinedione, troglitazone, in non-alcoholic steatohepatitis. Am J Gastroenterol 2001;96:519-25.

22. Renata B, Stephen A, Harrison. A placebo controlled trial of pioglitazone in subjects with non-alcoholic steatohepatitis. N Eng J Med 2006 ;355:2297-2307.

23. Schaffner F, Thaler H. Non-alcoholic fatty liver disease. Prog Liver Dis 1986;8:283-98. 